Review began 12/15/2021 Review ended 01/03/2022 Published 01/04/2022

๑) Copyright 2022

Alfaraj et al. This is an open access article distributed under the terms of the Creative Commons Attribution License CC-BY 4.0. which permits unrestricted use, distribution, and reproduction in any medium, provided the original author and source are credited.

\section{Pathological Fracture as the Initial Presentation of Metastatic Pancreatic Cancer}

\author{
Dunya Alfaraj $^{1}$, Rawan O. AlOtaibi ${ }^{2}$, Reema M. Bamousa ${ }^{2}$, Jana F. Alzamami ${ }^{2}$ \\ 1. Emergency Department, Imam Abdulrahman Bin Faisal University, King Fahad University Hospital, Dammam, SAU \\ 2. Medicine, Imam Abdulrahman Bin Faisal University, Dammam, SAU
}

Corresponding author: Rawan O. AlOtaibi ,rawan.omh@gmail.com

\begin{abstract}
Pancreatic cancer with bone metastasis is considered a very rare malignancy. Although the incidence rate of people with pancreatic cancer who develop bone metastasis is unknown, it is estimated as between $5 \%$ and $20 \%$. We report a 61-year-old lady who had multiple emergency department visits complaining of lower back pain radiating to the lower limbs for five months. Clinical and imaging workup suggested pancreatic
\end{abstract} adenocarcinoma metastasis to the second lumbar vertebra (L2), lungs, and ovaries.

Categories: Emergency Medicine, Oncology, Orthopedics

Keywords: pancreatic adenocarcinoma, pancreatic cancer, spinal fracture, pathological fractures, skeletal metastasis

\section{Introduction}

Pancreatic cancer accounts for about 3\% of all cancer in the United States and is about $7 \%$ of the cause of mortality among cancers worldwide, with a lifetime risk of about 1 in 64 [1]. The most common sites of metastases in pancreatic cancer are the liver and peritoneum. Other less common sites include the lung, brain, kidney, and bone [2-3]. Although the prevalence rate of skeletal metastases in patients with pancreatic cancer is unknown, it is estimated to be between 5\% and 20\% [3]. According to the American Cancer Society, the five-year relative survival rate in metastatic pancreatic cancer patients is about 3\% [4]. In Italy, a case of pancreatic cancer was reported with vertebral fracture and spinal cord compression [5]. In China, they reported a case of pancreatic cancer with a vertebral compression fracture [6]. In Athens, vertebral metastasis was found in a case of pancreas cancer with back pain as an initial presentation [3]. In another case reported in Japan, they found right iliac bone and spinal metastases in a patient with pancreatic cancer [7]. Furthermore, in the USA, they reported a case of pancreatic cancer with right clavicular head metastases [8]. One more case in the USA reported sacrum metastasis as the only site in a patient seven years after treatment for a locally advanced pancreatic adenocarcinoma [9].

\section{Case Presentation}

Initially, a 61-year-old Saudi female patient with a known case of controlled hypertension came to the emergency department (ED) complaining of low back pain that started one day ago after she slept; however, she hadn't suffered a fall. The pain was exacerbated by movement, not radiating to the lower limbs, and not associated with any neurological defects. Physical and neurological examinations were unremarkable. In addition, an X-ray of the lumbar spine was done (Figure 1) and no findings were reported so the patient was discharged on conservative management. A few weeks later, she had several visits to the ED with the same complaint, and her pain was increasing, which made her unable to sit or lie supine for long periods. A decreased range of motion of the spine was noticed, but no numbness or weakness was reported. The general physical examination was normal, and the lower limb neurological examination showed bilateral hip flexion power of four out of five at the L2 level, positive right side Babinski sign, and bilateral intact sensation at all levels. During the same visit, an X-ray of the lumbar spine was done (Figure 2) as well as a CT of the lumbar spine, which showed an L2 vertebral body compression fracture with no definite compression on the spinal cord or retropulsion fragment and normal CT features of the paravertebral soft tissues (Figure 3). However, since the patient's condition improved, she was discharged from the ED and was referred to the orthopedic spine clinic for follow-up. 


\section{Cureus}

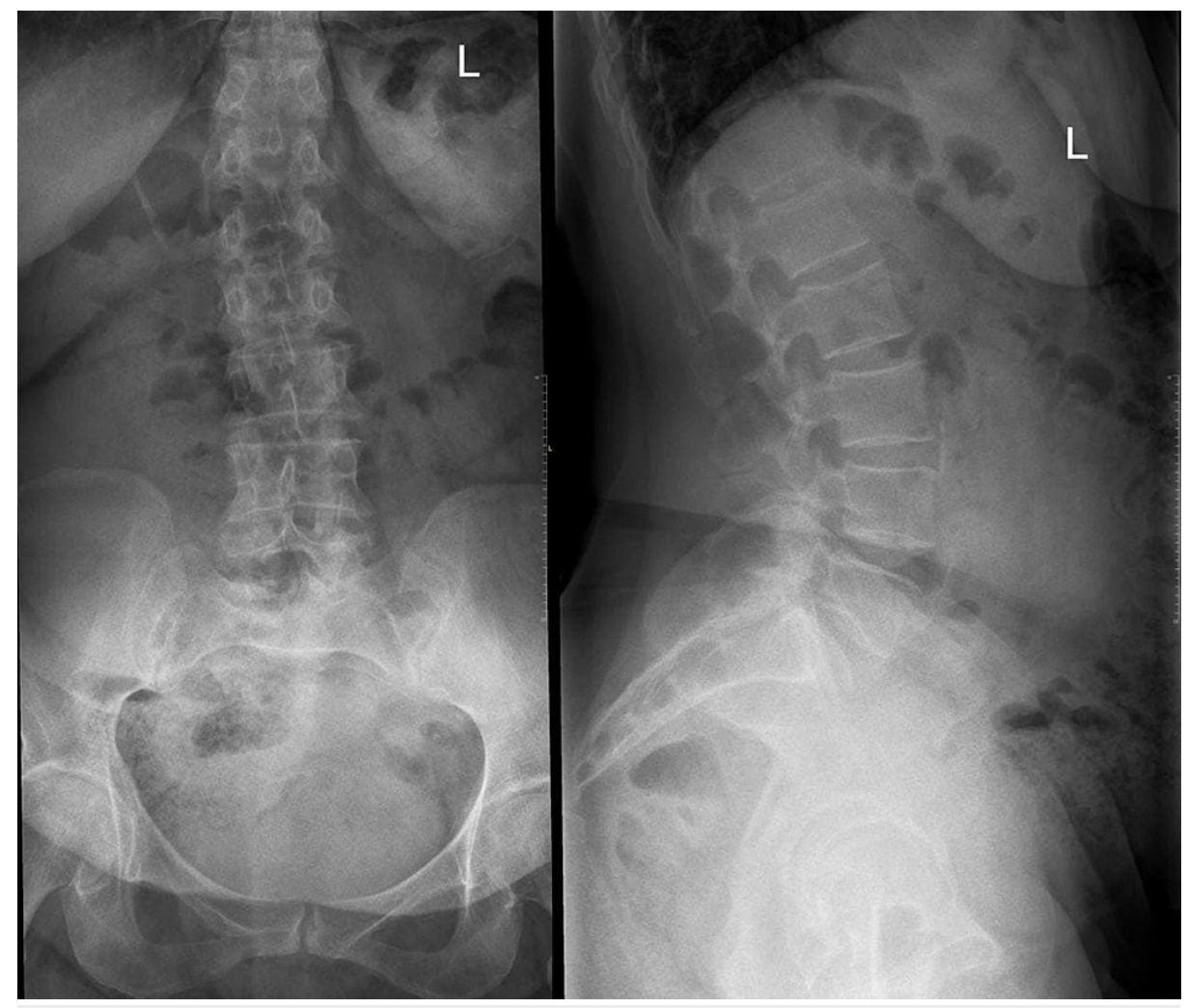

FIGURE 1: X-ray of the lumbar spine; anterior-posterior (AP) and lateral views

Showing unremarkable lumbar spine 


\section{Cureus}

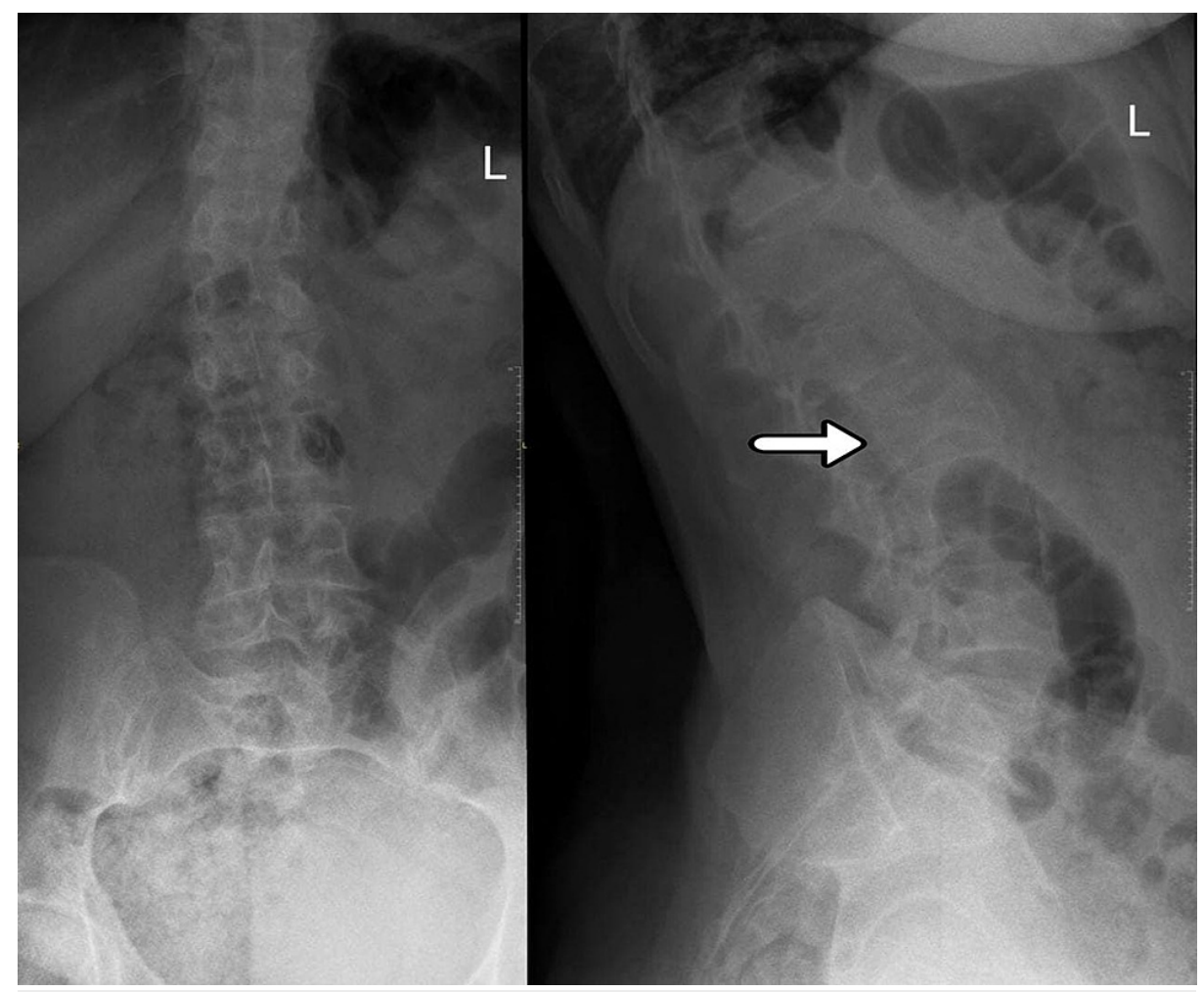

FIGURE 2: X-ray of the lumbar spine; AP and lateral views

Showing L2 compression, with no obvious fracture 


\section{Cureus}

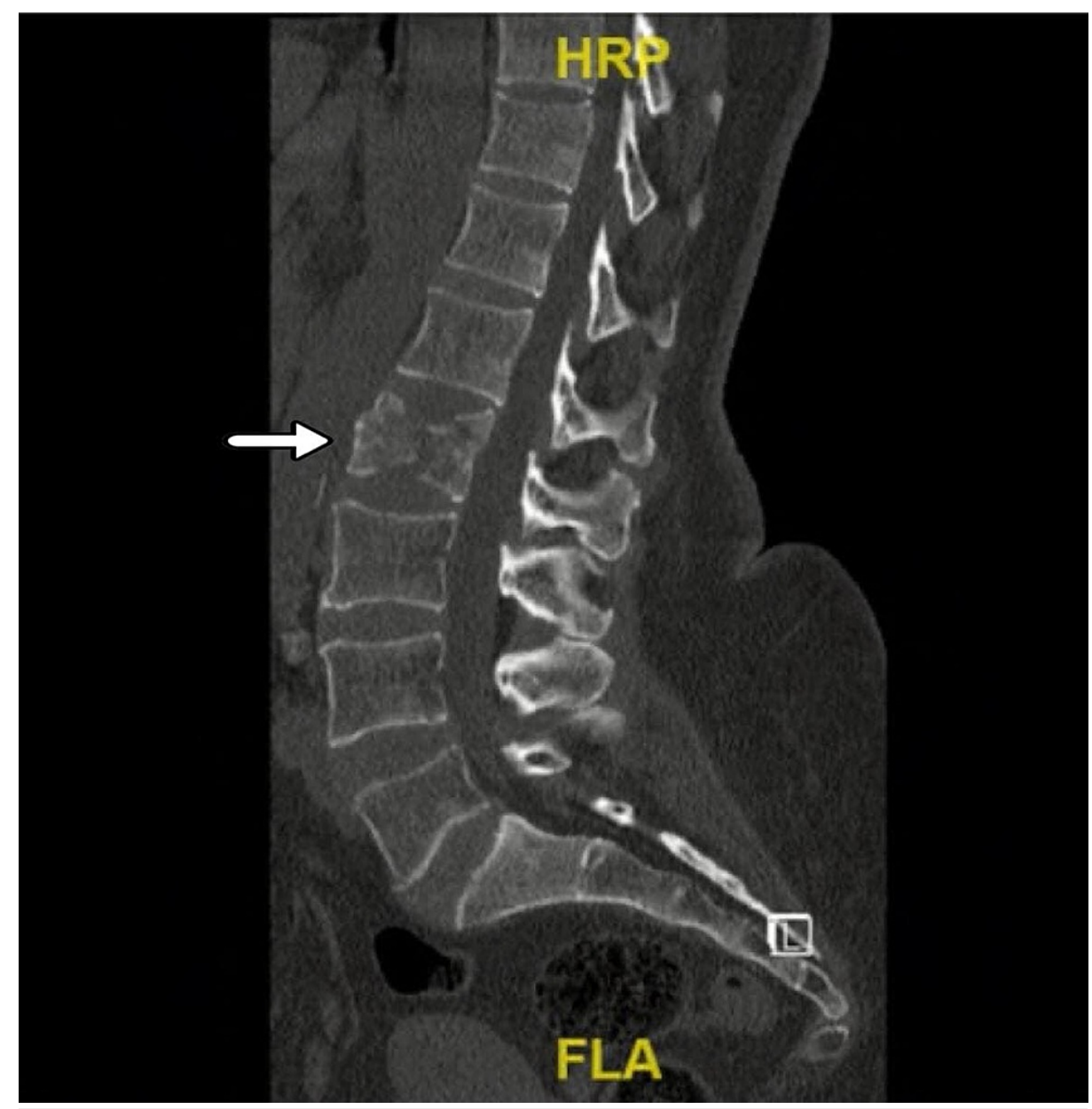

\section{FIGURE 3: CT scan lumbar without contrast}

Showing L2 vertebral body compression fracture with no definitive compression on the spinal cord

The patient was following up regularly with the orthopedic spine clinic and had multiple ED visits before admission. However, three months after her first ED visit, the patient was admitted through the outpatient clinic for further investigations since her back pain was progressively increasing with no improvement. During the admission, a lumbar spine MRI (Figure 4) was done, which concluded an interval progression of L2 compression fracture with significant height reduction compared to the old CT that was done three months prior to her admission. Moreover, a paravertebral component and retropulsion were noticed that were compressing the cauda equina causing severe spinal canal stenosis and bilateral neural foraminal narrowing. 


\section{Cureus}

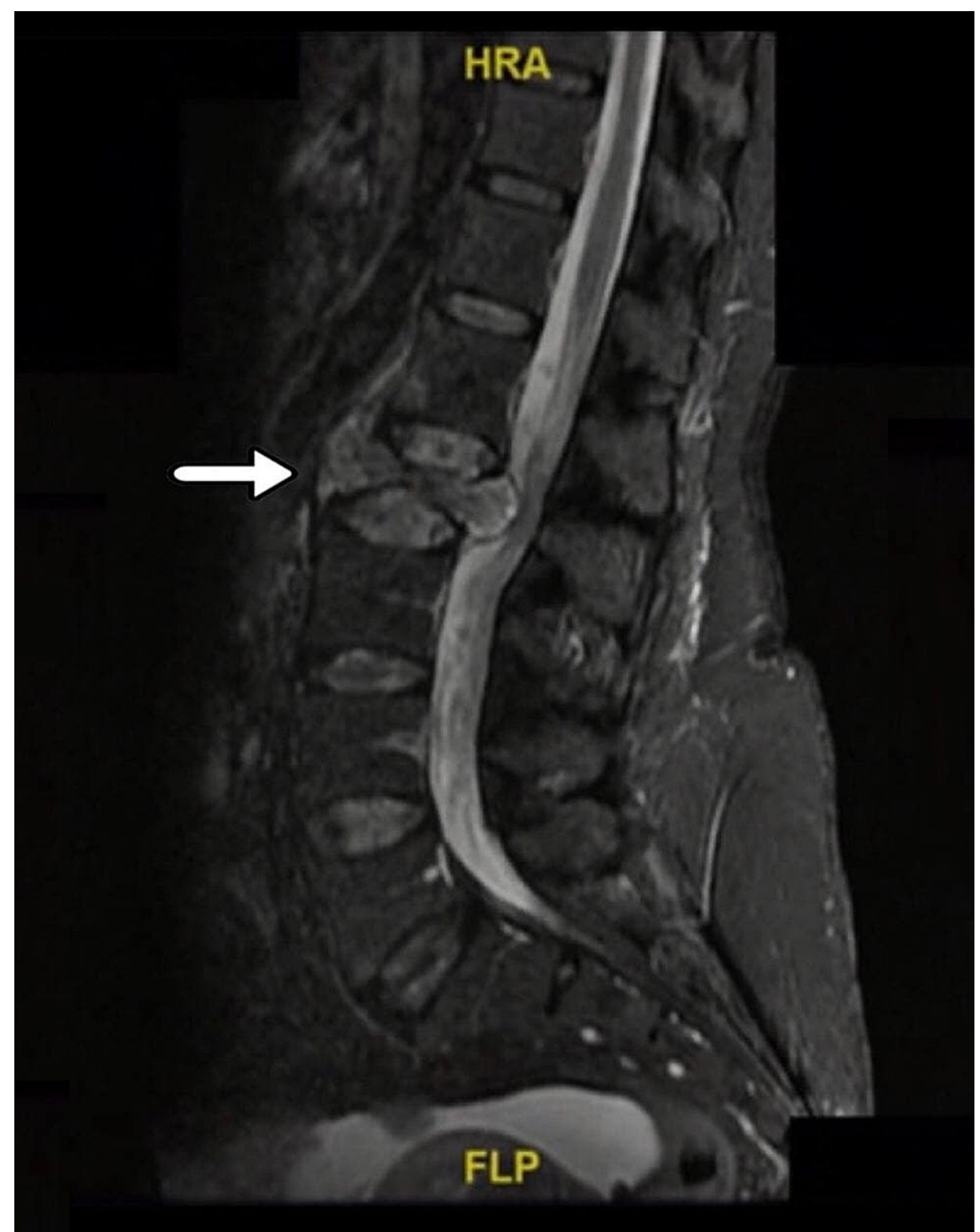

\section{FIGURE 4: MRI scan lumbar spine}

Showing L2 compression fracture with significant height reduction

She also underwent chest abdomen pelvis CT (CAP CT) scan, and multiple scattered pulmonary nodules were found as well as a poorly defined pancreatic tail hypodense mass measuring $3.9 \mathrm{~cm}$, bilateral complex cystic ovarian lesions were found most likely metastatic lesions, and overly distended gallbladder containing gallstones with no evidence of cholecystitis.

The patient was scheduled for posterior instrumented spinal fusion to regain spine stability and open pedicular biopsy from the vertebral body (L2). The gross histopathological description showed multiple tan/brown firm to hard tissue fragments measuring in aggregate $1.4 \mathrm{X} 1.2 \mathrm{X} 0.2 \mathrm{~cm}$ and the immunochemical panel results came out as the following: CK7 and CK19: Positive; CK20, PAX8, and TTF1: Negative. Moreover, the radiologic finding with the elevated tumor marker (CA 19-9) along with the histopathological and immunochemical profile are in favor of primary pancreatic origin.

The patient and her family were informed and educated about the diagnosis, prognosis, and treatment options on multiple sessions, and the patient was transferred to a tertiary hospital for further evaluation, management, and follow-up.

\section{Discussion}

Pancreatic cancer is considered the seventh cause of cancer-related deaths worldwide. It is described as an 
aggressive type of cancer that has a very poor prognosis and high mortality rates [1-10]. According to Torre et al. (2012), the incidence of pancreatic cancer in developed countries and less developed countries was found to be $1 \%$ and $0.4 \%$, respectively [10]. In addition, the five-year survival rate was found to be less than $5 \%$ in patients with pancreatic cancer [4-11]. The most common sites of pancreatic cancer metastasis are the liver and peritoneum. However, the brain, lungs, kidneys, and bones are considered unusual sites for metastasis [2-3]. According to Borad et al. (2009), the spine was the most common site of skeletal metastasis in the case of an advanced stage of pancreatic cancer. As back pain might be a symptom of pancreatic cancer itself, but it could be an indication of bone metastasis as well [2]. Usually, in the early stages of pancreatic cancer, patients would be asymptomatic. However, the diagnosis mostly depends on the symptoms, which indicates late stages and, thus, poor prognosis. In some cases, the pancreatic tumor is suspected due to a predisposing condition, which will lead to an early diagnosis and a better prognosis. The symptoms of pancreatic cancer include abdominal pain, anorexia, early satiety, insomnia, and weight loss. Moreover, back pain might be present, and it would indicate an unresectable tumor, poor prognosis, and low survival rates [12]. In patients with suspicion of pancreatic cancer, laboratory investigations, including complete blood count (CBC), liver enzymes to exclude biliary obstruction, fasting glucose, and glycated hemoglobin (HBA1C) to rule out diabetes. Patients with jaundice or nonspecific abdominal pain can initially undergo an ultrasound followed by CT if pancreatic cancer is suspected. Patients with contraindications of CT would undergo MRI. If imaging results are unclear, endoscopic ultrasound- or CT-guided biopsies can be used for further evaluations [13]. According to Puri et al. (2021), skeletal metastases in pancreatic cancer are considered uncommon, a sample included 207 patients who were diagnosed with advanced pancreatic cancer, during the evaluation of these patients, only 19 (9.2\%) were detected with skeletal metastasis. The thoracic and lumbar spine was the most common site for skeletal metastasis. The initial observed symptom was bone pain in seven out of 19, pathological fractures were two out of 19, and one out of 19 patients had inferior vena cava compression secondary to para-spinal mass [14]. In our case, the patient presented with low back pain and decreased range of motion due to compression fracture of the spine, the patient underwent lumbar spine CT, which showed L2 compression due to metastasis. After further workup patient was diagnosed incidentally with pathological metastatic adenocarcinoma. Surgical resection of the tumor is considered the only curative management for pancreatic cancer. However, most of the patients will present with locally advanced unresectable lesions. Some studies suggested the use of chemoradiation therapy to convert the unresectable lesions to surgically resectable lesions, which eventually will have the same prognosis as patients who were diagnosed initially with resectable lesions, but this method is still controversial [15]. In case of locally advanced lesions with metastasis, the National Comprehensive Cancer Network recommends starting with FOLFIRINOX or gemcitabine monotherapy to relieve the symptoms and slightly improve the survival rate (several months). Moreover, a combination of gemcitabine and erlotinib, capecitabine, or oxaliplatin can be used as the second-line therapy for advanced stages of pancreatic cancer [15-16]. However, radiation therapy is only indicated in patients who progress metastatic disease and are in need of palliative care [17]. Survival periods of pancreatic cancer patients with bone metastases are usually not long, however, treatment for bone metastases is important in terms of quality of life. An earlier diagnosis is essential to prevent deterioration in the quality of life of pancreatic cancer patients presenting with bone metastases [18]. In our case, our patient underwent an open pedicular biopsy from the vertebral body (L2). The pathology report confirmed the metastasis, the patient was informed about the pathology report and referred to a tertiary hospital for a further management plan.

\section{Conclusions}

There are limited examples of pathological fractures as the initial presentation of metastatic pancreatobiliary adenocarcinoma in the literature. This case illustrates the unusual dissemination of pancreatobiliary adenocarcinoma. However, elderly patients with bone pain and pathological fractures must be taken seriously and a full investigation must be done to rule out bone metastasis and malignancy before they reach an advanced stage. In conclusion, considering back pain and pathological fractures as bone metastasis will help in reaching the appropriate diagnosis and will give the patient appropriate care and, hopefully, a better prognosis.

\section{Additional Information Disclosures}

Human subjects: Consent was obtained or waived by all participants in this study. Conflicts of interest: In compliance with the ICMJE uniform disclosure form, all authors declare the following: Payment/services info: All authors have declared that no financial support was received from any organization for the submitted work. Financial relationships: All authors have declared that they have no financial relationships at present or within the previous three years with any organizations that might have an interest in the submitted work. Other relationships: All authors have declared that there are no other relationships or activities that could appear to have influenced the submitted work.

\section{References}

1. Lifetime risk (percent) of being diagnosed with cancer by site and race/ethnicity: both sexes, 18 SEER Areas, 2013-2015 (Table 1.15). (2018). https://seer.cancer.gov/csr/1975_2015/results_merged/topic_lifetime_risk.pdf. 
2. Borad MJ, Saadati H, Lakshmipathy A, et al.: Skeletal metastases in pancreatic cancer: a retrospective study and review of the literature. Yale J Biol Med. 2009, 82:1-6.

3. Pneumaticos SG, Savidou C, Korres DS, Chatziioannou SN: Pancreatic cancer's initial presentation: back pain due to osteoblastic bone metastasis. Eur J Cancer Care (Engl). 2010, 19:137-40. 10.1111/j.13652354.2007.00920.x

4. Survival rates for pancreatic cancer. (2021). https://www.cancer.org/cancer/pancreatic-cancer/detectiondiagnosis-staging/survival-rates.html.

5. Argentiero A, Calabrese A, Solimando AG, Notaristefano A, Panarelli MM, Brunetti O: Bone metastasis as primary presentation of pancreatic ductal adenocarcinoma: a case report and literature review. Clin Case Rep. 2019, 7:1972-6. 10.1002/ccr3.2412

6. Chih YP, Wu WT, Lin CL, Jou HJ, Huang YH, Chen LC, Chou LW: Vertebral compression fracture related to pancreatic cancer with osteoblastic metastasis. A case report and literature review. Medicine (Baltimore). 2016, 95:e2670. 10.1097/MD.0000000000002670

7. Ito H, Tajiri T, Hiraiwa SI, et al.: A case of rare cutaneous metastasis from advanced pancreatic cancer . Case Rep Oncol. 2020, 13:49-54. 10.1159/000505322

8. Saif MW, Galanina N, Ravage-Mass L, Kaley K, Cornfeld D, Lamb L, Chhieng D: Bone metastasis as the only metastatic site in a patient with pancreatic cancer following distal pancreatectomy. Case Rep Med. 2010, 2010:634975. 10.1155/2010/634975

9. Ray AE, Faltings L, Machnicki S, et al.: Bone metastasis as the only site of disease in a patient 7 years post treatment for a locally advanced pancreatic adenocarcinoma. J Pancreas. 2018, 19:296-302.

10. Torre LA, Bray F, Siegel RL, Ferlay J, Lortet-Tieulent J, Jemal A: Global cancer statistics, 2012. CA Cancer J Clin. 2015, 65:87-108. 10.3322/caac.21262

11. Lunardi S, Muschel RJ, Brunner TB: The stromal compartments in pancreatic cancer: are there any therapeutic targets?. Cancer Lett. 2014, 343:147-55. 10.1016/j.canlet.2013.09.039

12. DiMagno EP: Pancreatic cancer: clinical presentation, pitfalls and early clues . Ann Oncol. 1999, 10:S140-2. 10.1093/annonc/10.suppl_4.s140

13. Kanji ZS, Gallinger S: Diagnosis and management of pancreatic cancer. CMAJ. 2013, 185:1219-26. 10.1503/cmaj.121368

14. Puri A, Chang J, Tanner N, et al.: Skeletal metastases in advanced pancreatic ductal adenocarcinoma: a retrospective analysis. J Gastrointest Oncol. 2021, 12:455-63. 10.21037/jgo-20-361

15. National Comprehensive Cancer Network. NCCN clinical practice guidelines in oncology NCCN. Pancreatic adenocarcinoma. Version 2. (2021). Accessed: December 26, 2021: https://www.poijaya.org/wpcontent/uploads/2021/08/pancreatic.pdf.

16. Gourgou-Bourgade S, Bascoul-Mollevi C, Desseigne F, et al.: Impact of FOLFIRINOX compared with gemcitabine on quality of life in patients with metastatic pancreatic cancer: results from the PRODIGE 4/ACCORD 11 randomized trial. J Clin Oncol. 2013, 31:23-9. 10.1200/JCO.2012.44.4869

17. Quiros RM, Brown KM, Hoffman JP: Neoadjuvant therapy in pancreatic cancer. Cancer Invest. 2007, 25:26773. 10.1080/07357900701206356

18. Iguchi H, Yasuda M, Matsuo T, Sumii T, Funakoshi A: Clinical features and management of pancreatic cancer with bone metastases [Article in Japanese]. Nihon Shokakibyo Gakkai Zasshi. 2004, 101:872-8. 